\title{
THE ERGONOMICS OF SYRINGE OPERATION DURING THE INJECTION OF FLUID INTO TISSUE EXPANDERS
}

\author{
Sarah A. Horton, Marc Doumit \\ Department of Mechanical Engineering \\ University of Ottawa \\ Ottawa, ON, Canada \\ marc.doumit@uottawa.ca
}

\author{
Gloria Rockwell \\ Division of Plastic Surgery Ottawa Hospital General \\ University of Ottawa \\ Ottawa, ON, Canada \\ office@plasticsurgerymd.ca
}

\begin{abstract}
The reconstruction process following a mastectomy procedure may require the use of a tissue expander(s) to accommodate an implant. Over several months, the expanders are routinely filled with sterile saline solution to gradually stretch the skin and muscle around the subpectoral pocket. The saline solution is injected manually by the surgeon or nurse into the expander using a syringe, catheter and needle. The injection process is physically demanding and repetitive; it has been reported to pose negative long-term effects on the muscles and joints of the hand, causing repetitive strain injuries for the syringe operator. Thus, the purpose of this study is to analyze the injection process and provide a comprehensive understanding of the factors that can be intervened upon to make this process more ergonomic and safe. To achieve this understanding, a laboratory testing setup is developed to mechanically simulate and analyze the fluid injection process into tissue expanders. Experimental results show that the magnitude of the syringe force required to inject the fluid is significantly correlated to the rate of compression, the size of the syringe and the resistance produced by the stretching of tissue expander. Moreover, the magnitude of forces measured during testing are found to be well above the recommended values to prevent repetitive stress injuries.
\end{abstract}

Keywords- Breast Tissue Expander, Breast Implant, Biomechanics, Ergonomics, Mastectomy.

\section{INTRODUCTION}

The mastectomy procedure is a common prophylactic management for breast cancer, wherein some or all the breast tissue and overlying skin is removed. The breast implant involves the insertion of a synthetic sac-like prosthesis under the skin and muscle structures of the chest wall to mimic the natural breast mound. In this process, a tissue expander can be utilized prior to implant insertion to reduce discomfort and maximize the aesthetic outcome. The expander is a flexible shell that is inserted into the subpectoral pocket immediately postmastectomy or later. It is gradually injected with a saline solution, which increases the volume of the expander shell to create a breast mound, and consequently stretch the involved skin and muscle tissue. Saline solution is introduced through the skin of the patient into the expander through a syringe, catheter and needle, via a fill port located within the expander [1]. Figure 1 shows these elements.

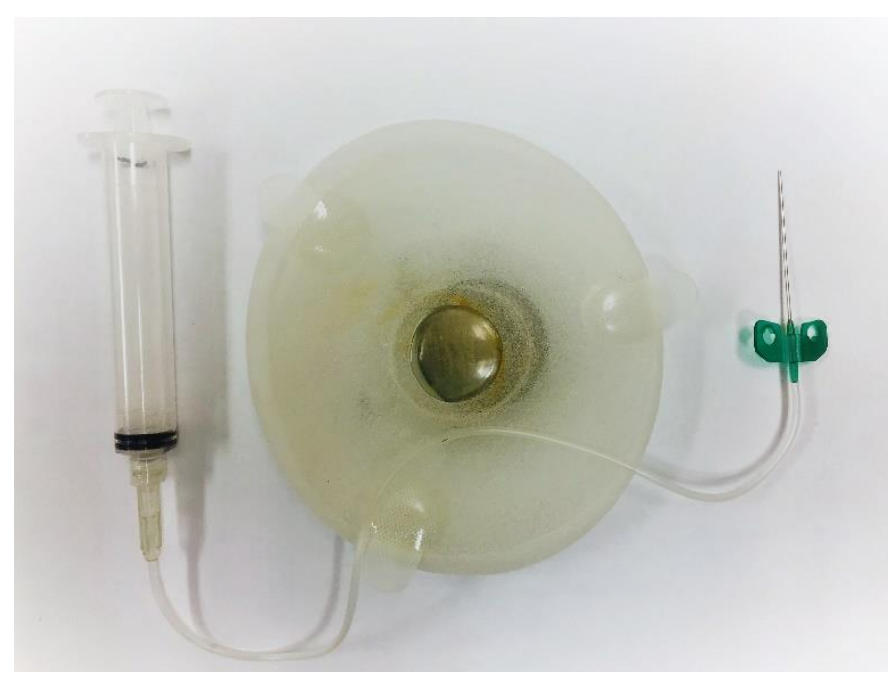

Figure 1: Syringe, catheter, needle and breast implant expander

Repetitive hand strain injuries are an occupational hazard that can cause long-term disability and impact quality of life of operators. Actions such as applying force to a syringe plunger for a prolonged period when filling a tissue expander can cause joint pain and even cause cumulative trauma disorders, such as carpal tunnel syndrome [2]. It is believed that the trauma is inflicted by repetitive tension in the flexor tendons of the hand, which over time lead to inflammation and compression of the median nerve within the carpal tunnel [3].

The force applied on a syringe by the operator resembles a chuck pinch, or a pinch implicating the thumb, the second and third digits. A study conducted on eight female and eight male participants found that a thumb in hyperextended position can achieve a maximum pinch force of $49.49 \mathrm{~N}$ and $73.7 \mathrm{~N}$, respectively, when the wrist was in a neutral position [4-5]. However, pinch strength appears to decrease significantly, as the wrist is flexed as well as with the use of the non-dominant hand 
[6-7]. Moreover, if the thumb is not hyperextended, the resulting force is inferior and pinch force is inversely proportional to the pinch width [8]. Other studies also reported that the maximum acceptable force for tasks completed two times per week at a rate of two repetitions per minute involving repetitive flexion pinch grips is $6.35 \mathrm{~N}$ [9], which is considerably less than the maximum pinch force abilities of the human hand. This is comparable to the frequency that a surgeon or nurse operates a syringe during the expansion procedure on a weekly basis.

Through the literature review, no studies have analyzed the mechanical behaviour of the injection process for tissue expanders and its human factor implications. Consequently, it is unclear to what extent different factors germane to the expander expansion process can influence comfort and ergonomics for the operator. Thus, this study aims to achieve a comprehensive understanding of this mechanical procedure and determine the optimal ergonomic method for filling the breast tissue expanders.

\section{METHOD}

In this study, the mechanical behavior of tissue expanders during the expansion process is experimentally characterized using an Instron ${ }^{\mathrm{TM}}$ universal testing machine. The magnitude of the syringe force required to inject the fluid into the expander is analyzed while altering key variables. Five variables that are believed to have an impact on the syringe injection mechanism and its ergonomics are selected and presented as follow:

\section{Syringe size:}

Different operators use distinct syringe sizes to inject fluid. A varying syringe size corresponds to a changing plunger surface area and thus a change in the force required to create the same fluid pressure. In this study, three common syringe sizes are tested to evaluate their impact: 10, 20 and $60 \mathrm{~mL}$.

\section{Syringe compression speed:}

The operator can only inject the fluid at a limited speed before feeling a substantial syringe force resistance. It is believed that the injection speed affects the plunger dynamics, fluid resistance through the needle and catheter as well as the resistance caused by the mechanical properties of the tissue expander. For this variable, syringe compression speeds of 20 , 25 and $30 \mathrm{~mm} / \mathrm{min}$ are selected and tested.

\section{Expander size:}

The size of the tissue expander varies between patients such to adapt to their needs. A different expander size equates to a different volume and surface and thus alters the resistive behavior of the tissue expander especially when inserted in the subpectoral pocket. For these tests, expanders from Naturelle [10] in common volume sizes of 250, 300, 400, 500, 600, 700, 750 and $850 \mathrm{~mL}$ are selected and tested.

\section{Expander resistance:}

The skin and muscle of the patient creates a resistance against the expansion process. The pressure applied to the tissue expander is hypothesized to restrict the flow of fluid coming from the syringe, thus increasing the required syringe force applied by the operator. Two constant weights of 1 and $2 \mathrm{lbs}$ are placed on the tissue expanders while testing to simulate constant skin resistance load.

\section{Injected fluid volume:}

A limited amount of fluid may be injected per visit to minimize discomfort. Expanders with larger volumes tend to have a larger diameter, which may cause an unsymmetrical or incorrect breast shape for patients with smaller chest widths. In these cases, smaller expanders with the correct diameter are overfilled (filling the expander beyond the recommended fluid amount) to further stretch the skin and give a more natural appearance. Consequently, this process amplifies the effort required to inject the saline solution and leads to hand fatigue and discomfort. This scenario is mechanically simulated to analyze the extent of the impact of filling the expander past the rated volume.

To achieve the tests and evaluate the impact of the five selected variables, an experimental apparatus is developed in laboratory to facilitate standardized measurements of the tissue expanders expansion mechanism. As shown in Figure 2, a supporting jig is fabricated using a PVC tube with three openings: one at the top to observe the amount of liquid dispensed, and two at opposite sides of the tube's base to facilitate access to the expanders, catheter and needle. A plexiglass circle with a centered hole was attached to the top of the tube using mechanical fasteners to accommodate the placement of the syringes. The expanders were elevated to help keep the catheter straight and minimize additional resistance.

All data is collected using an Instron ${ }^{\mathrm{TM}}$ universal testing machine with a $1 \mathrm{kN}$ load cell with an accuracy of $+/-0.25 \%$. Tap water is used as a substitute to saline fluid for simplicity.

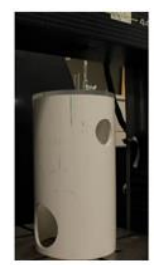

a)

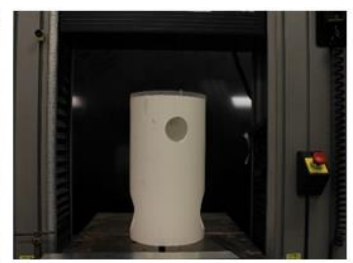

b)

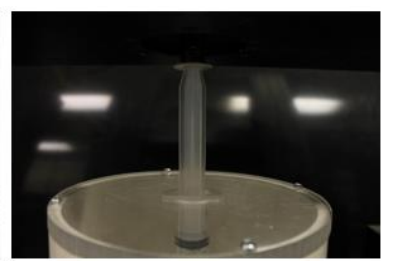

c)
Figure 2: (a) Front view b) Side view c) Top view of the plastic tube (with $60 \mathrm{~mL}$ syringe)

\section{RESULTS}

The introduction of the five selected variables throughout testing had shown a substantial impact on the syringe force required to inject the fluid into the tissue expanders. A first test is conducted, without the use of a tissue expander where the fluid is simply injected into an open container. This is achieved to determine the mechanical behaviour attributed to the syringe plunger, catheter and needle mechanisms. The data from this test is presented in Figure 3. This graph also shows a general profile of the curve found in all the achieved tests. The curve displays the transient and steady state response of the syringe force (yaxis, load) when the plunger is moved by the Instron machine with a uniform displacement speed. For clarity and simplicity, only the average of the syringe force will be presented as a bar plot for all subsequent presented results. 


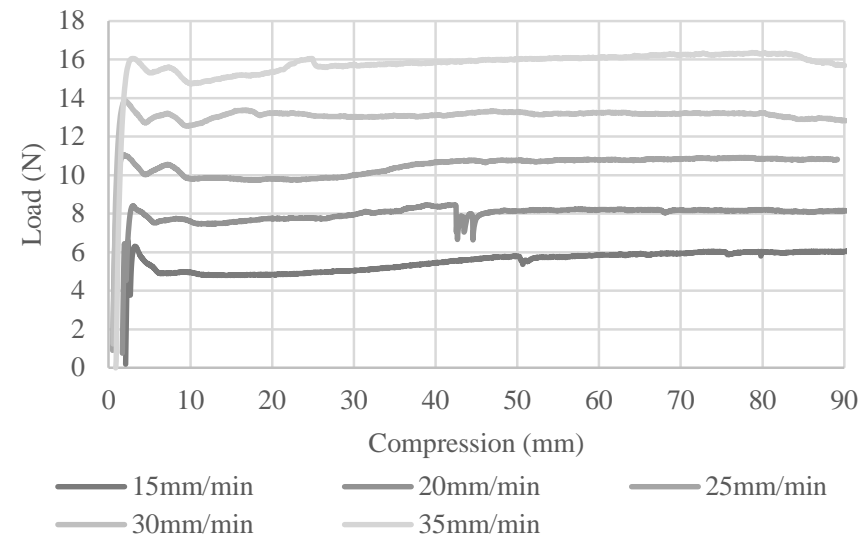

Figure 3: Compressive syringe force (Load) vs compression for a $60 \mathrm{~mL}$ syringe subjected to compression rates of $15,20,25,30$ and $35 \mathrm{~mm} / \mathrm{min}$

Preliminary experimental tests showed that the results are relatively consistent as the tissue expander is progressively filled until it reaches its recommended volume (without being overfilled). Thus, the following results and analysis will only consider the overfill scenarios such to demonstrate the effects of this practice on the required syringe force.

\section{Syringe size and syringe compression speed rate}

Figure 4 shows the results for the average compression force for three distinct syringe sizes $(10,20$ and $60 \mathrm{~mL})$ while varying the compression speed rate $(20,25$ and $30 \mathrm{~mm} / \mathrm{min})$. The test is achieved using a $250 \mathrm{~mL}$ expander and being overfilled with an additional volume of $60 \mathrm{~mL}$.

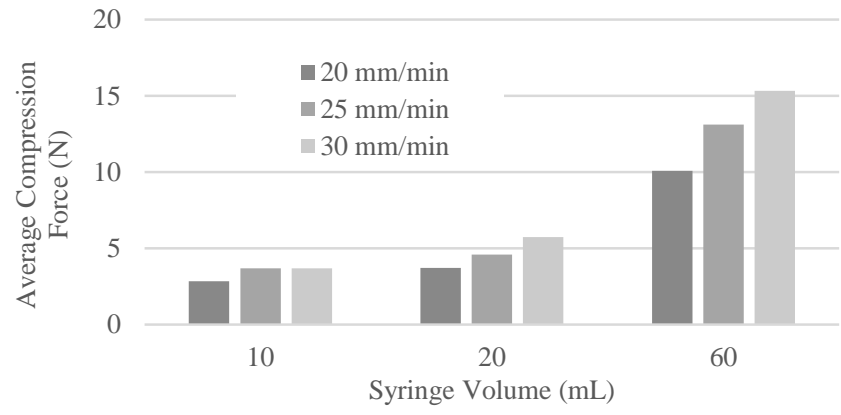

Figure 4: Average compression force of various syringe sizes subjected to a varying compression rate using a $250 \mathrm{~mL}$ expander

\section{Expander size}

Figure 5 shows the results for the average compression force for eight distinct tissue expanders $(250,300,400,500,600,700$, $750,850 \mathrm{~mL}$. The tests are conducted at a speed of $25 \mathrm{~mm} / \mathrm{min}$ while being overfilled with an additional volume of $60 \mathrm{~mL}$ using a $60 \mathrm{~mL}$ syringe.

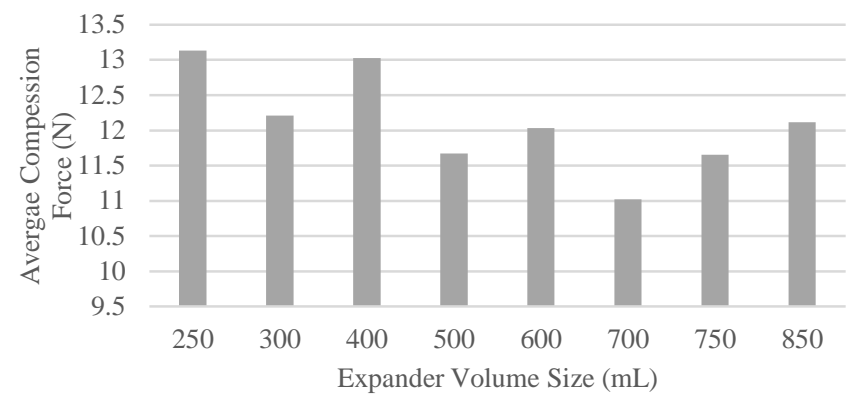

Figure 5: Average compression force for a $60 \mathrm{~mL}$ syringe subjected to various expander sizes at a compression rate of $25 \mathrm{~mm} / \mathrm{min}$

\section{Expander resistance}

Figure 6 shows the results for the average compression force for three distinct resistance weights (1,2 and $3 \mathrm{lbs}$.). The tests are conducted at a speed of $25 \mathrm{~mm} / \mathrm{min}$ while being overfilled with an additional volume of $60 \mathrm{~mL}$ using a $60 \mathrm{~mL}$ syringe.

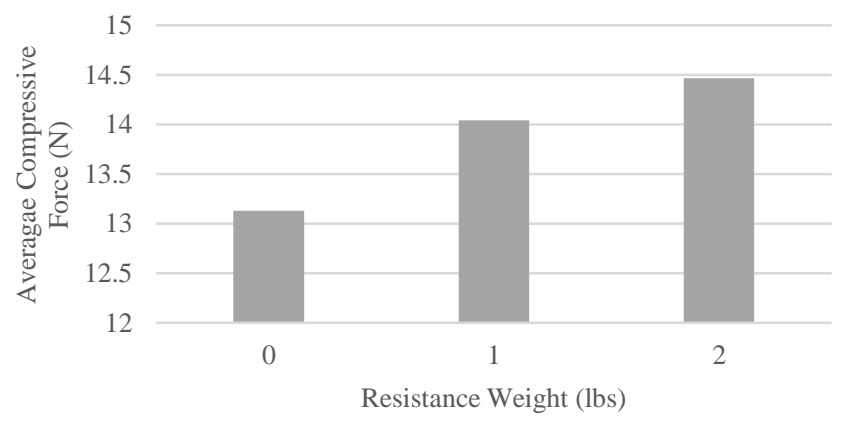

Figure 6: Average compression force for a $250 \mathrm{~mL}$ expander subjected to various exterior resistance with a compression rate of $25 \mathrm{~mm} / \mathrm{min}$ using a 60 $\mathrm{mL}$ syringe

\section{Injected fluid volume}

Figure 7 shows the average compression force for the seven sequences that are required to fill a $250 \mathrm{~mL}$ expander using a 60 $\mathrm{mL}$ syringe at a speed rate of $25 \mathrm{~mm} / \mathrm{min}$.

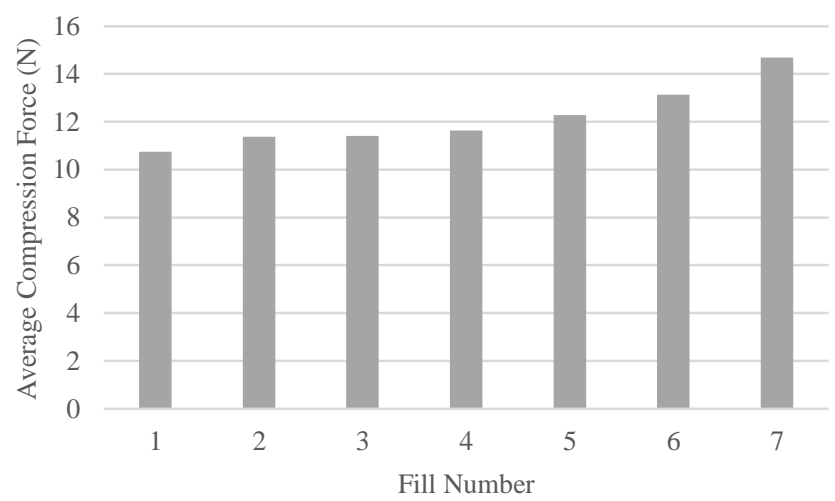

Figure 7: Average compression force of the seven sequences required to fill a $250 \mathrm{~mL}$ expander with a $60 \mathrm{~mL}$ syringe at a compression rate of $25 \mathrm{~mm} / \mathrm{min}$

\section{Discussion}

The first results presented in Figure 3 display the transient and steady state response of the syringe force upon the Instron 
machine moving the syringe plunger with a uniform compression speed. Depending on the speed, the measured force varied between 5 and $16 \mathrm{~N}$ for a speed range of 15 to $35 \mathrm{~mm} / \mathrm{min}$. These represent significant force magnitudes given the absence of the tissue expander and patient skin resistance properties in these tests. Moreover, these results also demonstrate the viscoelastic behaviour of the syringe, catheter and needle mechanism.

\section{Syringe size}

The impact of syringe size on the compression force is demonstrated in Figure 4. It has been shown that even while varying the speed of compression, the magnitude of force measured remains proportional to the syringe size. Relatively low force magnitudes of $5 \mathrm{~N}$ or less were measured for the syringes of 10 and $20 \mathrm{~mL}$ while large force magnitudes of about $15 \mathrm{~N}$ were measured for the $60 \mathrm{~mL}$ syringe. Based on these results, a smaller syringe size would be ideal to achieve smooth and small syringe forces during the injection process. However, a smaller syringe size may be less desirable since it prolongs the expansion process and consequently the overall surgical procedure.

\section{Syringe compression speed}

Compression speed refers to the rate at which the syringe plunger is compressed $(\mathrm{mm} / \mathrm{min})$. Figure 4 shows how the compression speed affects the required compressive force while the rate varies from 20 to 25 to $30 \mathrm{~mm} / \mathrm{min}$. On average, a 5 $\mathrm{mm} / \mathrm{min}$ increase in speeds equates to a force increase of $2.65 \mathrm{~N}$ without an expander, and $2.54 \mathrm{~N}$ with an expander. The results demonstrate high viscoelastic behaviour and such when the compressive speed is increased; the compression force is also increased. Therefore, the use of a high compressive force to drive the syringe faster is counterproductive as intuitively experienced by operators. A slower and consistent syringe compression speed results in a smaller compression force and a safer process for the operator.

\section{Expander size}

Figure 5 shows the compression force while filling various expander sizes $(250,300,400,500,600,700,750,850 \mathrm{~mL})$. Given the absence of the skin resistance properties, there is no clear correlation between the compression force and the expander size. However, the two largest forces were found when filling the smaller expanders $(13.13 \mathrm{~N}$ for the $250 \mathrm{~mL}$ expander and $13.03 \mathrm{~N}$ for the $400 \mathrm{~mL}$ expander). Some limitations of the study may provide an explanation for this phenomenon.

\section{Expander resistance}

As seen in Figure 6, an increase in the external resistance simulated by the addition of the free weights (1 and $2 \mathrm{lbs}$.) resulted in an increase in the magnitude of the compression force. The addition of the $1 \mathrm{lbs}$. weight increased the average compression force by $0.91 \mathrm{~N}$, whereas the $2 \mathrm{lbs}$. weight caused an increase of $1.34 \mathrm{lbs}$. Similar but amplified resistance is expected by the skin and over-lying tissues given its nonuniform and increasing stiffness properties throughout deformation. The procedure may be more strenuous on patients with lower tissue elasticities (i.e. patients that have undergone radiation).

\section{Injected fluid volume}

Figure 7 shows data spanning the entire process of filling one $250 \mathrm{~mL}$ expander with a $60 \mathrm{~mL}$ syringe, at a compression rate of $25 \mathrm{~mm} / \mathrm{min}$. Data is divided into seven sequences, wherein 50 $\mathrm{mL}$ of saline was injected per sequence. The first five sequences represented complete filling of a $250 \mathrm{~mL}$ expander, whereas the last two sequences recorded the effects of overfilling the expander. The compression force required to push the syringe plunger remains relatively the same as the expander is being filled, with a small gradual increase of $0.39 \mathrm{~N}$ on average. However, the force tends to increase more rapidly as it nears full capacity and when the expander is being overfilled. The force increases by $0.85 \mathrm{~N}$ between the $5^{\text {th }}$ and $6^{\text {th }}$ trials, and then by $1.55 \mathrm{~N}$ between the $6^{\text {th }}$ and $7^{\text {th }}$ trails.

\section{Limitations}

The substitution of tap water for saline may have affected the magnitude of the measured forces since saline solution is denser than tap water and would therefore require larger force. However, the magnitude of the force difference is considered negligible.

Instability of the weights on the expander during the experiment may have caused inconsistencies during data acquisition. The variation in the placement of the weights could also have an impact on the magnitude of the compression force.

The absence of a correlation between the compression force and the expander size during overfill could be explained by the selected overfill amount. The overfill volume was chosen to be a constant $100 \mathrm{~mL}$ for all the expanders. This means that during the overfill phase of the experiment, a $250 \mathrm{~mL}$ expander was overfilled by $40 \%$ whereas a $500 \mathrm{~mL}$ expander was only overfilled by $20 \%$. Therefore, the smaller the expander, the higher the percentage of overfill. Since a higher percentage of overfill creates more pressure in the expander, it increases the magnitude of the compression force (as seen in the high force observed for the $250 \mathrm{~mL}$ and $400 \mathrm{~mL}$ expander in Figure. 5).

\section{CONCLUSION}

The measured magnitudes of force required to fill a breast tissue expander in a mechanical setup were mostly above the recommended value for preventing repetitive stress injuries given in [9]. The data showed quantifiable trends among most of the selected tested variables. It was observed that a slower compression speed required a smaller compressive force and was a more ergonomic alternative. The magnitude of the compressive force also decreased when the size of the syringe decreased. Therefore, a smaller syringe is more ergonomic as opposed to a larger one. An array of expander sizes was tested; however, the size of the expander did not display any definite effect on the magnitude of the compressive force. External resistance was also applied to the expander using free weights to mimic the resistance applied on the expander by the skin in a real case scenario. As the magnitude of the external resistance was increased, the magnitude of the compressive force also increased. Incorporation of the observed principles from this study (i.e. smaller syringe sizes and a slower rate of compression) into clinical practice may influence the incidence of repetitive strain injuries for surgeons and nursing staff who participate in tissue expansion on a routine basis. Other factors 
such as the use of a larger needle could facilitate the task but may cause additional discomfort to the patient. Further clinical research is needed to determine the significance of these adjustments.

\section{REFERENCES}

[1] S. Ahmed, A. Snelling, M. Bains, and I. H. Whitworth,"Breast reconstruction," BMJ, vol. 330, no. 7497, pp.943-948, Apr. 2005.

[2] V. Putz-Anderson, Cumulative Trauma Disorders. A Manual for Musculoskeletal Disorders of the Upper Extremity. London: Taylor \& Francis, 1988.

[3] E. M. Smith, D. A. Sonstergard, and W. H. Anderson, "Carpal tunnel syndrome: contribution of flexor tendons.," Arch. Phys. Med. Rehabil., vol. 58, no. 9, pp. 379-385, Sep. 1977.

[4] P. G. Dempsey and M. M. Ayoub, "The influence of gender, grasp type, pinch width and wrist position on sustained pinch strength - ScienceDirect," Science Direct, Mar-1996. [Online]. http://www.sciencedirect.com/science/article/pii/0169814194001081.

[Accessed: 14-Jul-2017].

[5] R. Terrell and J. L. Purswell, "The Influence of Forearm and Wrist Orientation on Static Grip Strength as a Design Criterion for Hand Tools," Proc. Hum. Factors Soc. Annu. Meet., vol. 20, no. 1, pp. 28-32, Jul. 1976.

[6] J. E. Fernandez, J. B. Dahaian, C. A. Halpern, and V. Viswanath, "The effect of wrist posture on pinch strength," Proc Hum. Factors Soc. 35th Annu. Meet. St. Monica CA, pp. 748-752, 1991.

[7] A. Swanson B., I. B. Matev, and G. De Goot, "The strength of the hand," Bull Prosthet, no. Res 10.14, pp. 145-153, 1970.

[8] S. N. Imrhan and R. Rahman, "The effects of pinch width on pinch strengths of adult males using realistic pinch-handle coupling," Int. J. Ind. Ergon., vol. 16, no. 2, pp. 123-134, Aug. 1995.

[9] S. H. Snook, D. R. Vaillancourt, V. M. Cirello, and B. S. Webster, "Psychophysical studies of repetitive wrist flexion and extension," Ergonomics, vol. 38, no. 7, pp. 1488-1507, Jul. 1995.

[10] Natrellesurgeon.com, '133 Shaped Tissue Expanders', 2018. [Online]. Available: https://www.natrellesurgeon.com/shaped-tissue-expanders/133. [Accessed 14-Feb-2018]. 